\title{
Bloating and Distention in Irritable Bowel Syndrome: The Role of Visceral Sensation
}

\author{
ANURAG AGRAWAL, ${ }^{*}$ LESLEY A. HOUGHTON, ${ }^{*}$ RICHARD LEA, ${ }^{*}$ JULIE MORRIS, ${ }^{\star}$ BRIAN REILLY, ${ }^{\S}$ \\ and PETER J. WHORWELL* \\ ${ }^{*}$ Neurogastroenterology Unit, University of Manchester, Manchester; ${ }^{\ddagger}$ Department of Medical Statistics, Wythenshawe Hospital, Manchester; and § Department of \\ Medical Physics, Wythenshawe Hospital, Manchester, United Kingdom
}

Background \& Aims: Abdominal bloating is an extremely intrusive symptom of irritable bowel syndrome (IBS) that is not always accompanied by an increase in abdominal girth (distention), raising the possibility that these 2 features of the condition may not share a common pathophysiology. A number of mechanisms have been postulated for bloating and distention, but the role of visceral sensation, which is often abnormal in IBS, has not been previously investigated, and this study aimed to address this question. Methods: Abdominal girth measured by ambulatory abdominal inductance plethysmography and bloating severity was recorded over 24 hours in 39 IBS-constipation (ages, 18-73 years) and 29 IBS-diarrhea patients (ages, 20-59 years) meeting Rome II criteria. Within 1 week, rectal sensory thresholds were assessed with a barostat using the ascending method of limits and tracking. Results: IBS patients who suffered with bloating alone had lower thresholds for pain $(P=.005)$, desire to defecate $(P=.044)$, and first sensation $(P=.07)$ compared with those who had concomitant distention irrespective of bowel habit. When patients were grouped according to sensory threshold, hyposensitive individuals had distention significantly more than those with hypersensitivity $(P$ $=.001$ ), and this was observed more in the constipation subgroup. Static and dynamic compliance did not differ among any of the groups. Conclusions: The symptom of bloating alone is associated with visceral hypersensitivity, suggesting that the pathogenesis of bloating and distention may not be the same. Consequently, treatment approaches may have to be different, and measuring visceral sensation could have utility in choosing the right therapeutic modality.

$\mathrm{T}$ he majority of patients with irritable bowel syndrome (IBS) complain of abdominal bloating, which characteristically varies in severity, increases during the course of the day, tends to get worse after meals, and usually subsides overnight. ${ }^{1,2}$ The symptom is particularly intrusive, with many individuals finding it more troublesome than their abdominal pain. ${ }^{3}$ In the past, it has been considered acceptable to regard bloating and distention as descriptors of the same phenomenon, but there is now accumulating evidence that this view is no longer tenable. Data originally derived from empirical studies $^{2}$ but recently supported by the use of more objective methods of investigation ${ }^{1}$ suggest that bloating accompanied by distention appears to be more commonly associated with IBS constipation (IBS-C) than with IBS diarrhea (IBS-D), thus providing evidence that, although closely related, these 2 terms may be describing subtly different pathophysiologic processes.

To investigate this problem further and more objectively, we have developed and validated a system called abdominal inductance plethysmography that allows abdominal girth to be accurately recorded over a 24-hour period in an ambulatory fashion. 4,5 Using this technique, we have shown that, in a group of IBS subjects, all of whom complained of bloating, only $48 \%$ exhibited distention as defined by an increase in abdominal girth beyond that observed in controls, which in some patients exceeded 10 cm. ${ }^{1}$

Over the years, a whole variety of mechanisms have been advocated to explain what might cause bloating, and this has now been further complicated by the possibility that distention may differ in some way. Factors that have been implicated include psychologic stress, deliberate protrusion of the abdomen, fluid retention, excessive gas production, abnormal gas handling, abnormal motility, anterior abdominal muscle weakness, and exaggerated abdominal accommodation reflex. ${ }^{6,7}$ However, visceral sensation has not been assessed in relation to bloating and distention, and this could be important because visceral hypersensitivity is a common finding in IBS, ${ }^{8,9}$ and it has even been suggested that this abnormality might be a biologic marker for the condition. ${ }^{8}$ In contrast, a smaller subset of patients exhibit visceral hyposensitivity, and this is usually associated with constipation. ${ }^{10,11}$ We have speculated that visceral hypersen-

\footnotetext{
Abbreviations used in this paper: IBS-C, irritable bowel syndrome constipation; IBS-D, irritable bowel syndrome diarrhea.

(c) 2008 by the AGA Institute 0016-5085/08/\$34.00 doi:10.1053/j.gastro.2008.02.096
} 
sitivity may be involved in the pathogenesis of bloating in the absence of distention, and it was the purpose of this study to investigate this question.

The aim of this study was to identify IBS patients complaining of bloating with or without associated distention and relate these findings to visceral sensation as determined by a rectal barostat. Distention was measured objectively using the technique of abdominal inductance plethysmography, and bloating was subjectively scored by the patient using a Lickert scale.

\section{Patients and Methods}

\section{Patients}

Sixty-eight patients with IBS, 39 with IBS-C, and 29 with IBS-D (age range, 18-73; mean, 37.4 years; 63 females) who satisfied the ROME II criteria ${ }^{12}$ and suffered from bloating as part of their symptom complex were recruited from the outpatient department of University Hospital of South Manchester NHS Foundation Trust. All but 6 patients (4 IBS-C and 2 IBS-D) in the study group were barostat naive. Forty-five age- and sexmatched healthy volunteers (age range, 20-67; mean, 35.1 years; 42 females) were also included in the study. All subjects underwent appropriate investigations to rule out organic gastrointestinal disease and did not exhibit a functional disorder of the upper gastrointestinal tract that was more prominent than their IBS symptomatology. All subjects drank below the recommended limit of alcohol and were not taking medications that might affect gastrointestinal function for at least 48 hours prior to taking part in the study.

\section{Abdominal Inductance Plethysmography}

The abdominal inductance plethysmography system has been described in detail in previous publications $^{4,5}$ and is based on the principle that a loop of wire forms an inductor, the inductance of which is dependent on the area enclosed by the loop. The wire is sewn into an elasticated fabric belt $8.5 \mathrm{~cm}$ wide in a zigzag fashion to allow for abdominal expansion (Respitrace inductive sensor; Ambulatory Monitoring Inc, New York, NY) and is worn in a belt-like fashion around the abdomen. Attached to the wire is a small electronic circuit unit, which incorporates an inductor in a resonant circuit whose output frequency varies with the area enclosed by the belt and a small battery operated microprocessor data logger, which records and stores the average frequency of the oscillator circuit for 30 seconds each minute. The data logger simultaneously records posture as lying, sitting, or standing via sealed mercury tilt switches (ASSEMtech Europe Ltd, Essex, UK) taped to the subject's thigh and chest. The cross-sectional area of the abdomen recorded by the equipment is then converted into a circumferential measurement.

\section{Protocol}

Following an overnight fast, all subjects attended the laboratory before $10 \mathrm{Am}$ to be fitted with the abdominal inductance plethysmography belt and returned 24 hours later to have it removed. This allowed objective measurement of abdominal girth and thus distention. During this time, each subject completed a hospital anxiety and depression questionnaire and was given a paper diary on which to record, at hourly intervals, the following parameters: severity of any subjective reports of abdominal bloating and pain/discomfort using a 6-point Lickert scale ( 0 , none; 1 , very mild; 2 , mild; 3 , moderate; 4 , severe; and 5, very severe), the time and consistency of any bowel movement, and the time and content of any food or drink consumed.

Sensory and motor responses to distention of the rectum were assessed within 1 week of the abdominal inductance plethysmography measurements in patients with IBS. On each occasion, the subject attended the laboratory at 9 AM after an overnight fast. Following a Fleet phosphate enema, a catheter (customized rectal barostat catheter, Part No. C7-2CB-R [22 French]; MUI Scientific, Mississauga, Ontario, Canada), to which was attached a polyethylene bag (Pillow Type Rectal Barostat Balloon, Part No. CT-BP600R; length, $22 \mathrm{~cm}$; diameter, $15 \mathrm{~cm}$; capacity, $600 \mathrm{~mL}$; MUI Scientific), was inserted into the rectum and positioned such that the center of the bag was $11 \mathrm{~cm}$ from the anal verge. The bag was then unfolded by transiently inflating it with $150 \mathrm{~mL}$ air and then deflating it completely (conditioning distention). After a 1-hour recovery period, the catheter was connected to a computer-driven volume displacement device (G \& J Electronics Inc, Toronto, Ontario, Canada), and the pressure in the bag was increased in increments of 2 $\mathrm{mm} \mathrm{Hg}$ until respiratory excursions were observed or, if respiratory variations were not obvious, set at $12 \mathrm{~mm} \mathrm{Hg}$ (basal operating pressure). This pressure was then maintained for 15 minutes while monitoring the intrabag volume continuously to give a measure of rectal tone.

The bag was then serially inflated from the basal operating pressure in increments of $4 \mathrm{~mm} \mathrm{Hg}$ until moderate pain was experienced (see below) or a maximum pressure of $48 \mathrm{~mm} \mathrm{Hg}$ was reached (ascending method of limits). Each inflation was maintained for 2 minutes, and separated by 2 minutes, during which the pressure in the bag was returned to the basal operating pressure. Sixty seconds after commencement of each inflation, the patients were prompted to indicate on a standard chart whether they were experiencing pain/discomfort on the following scale: 0 , no pain/discomfort; 1 , mild but not sustained pain/discomfort; 2, mild and sustained pain/ discomfort; 3 , moderate pain; 4 , intense pain. When the subject first experienced moderate pain (score, 3), the volume displacement device was programmed to terminate the ascending method of limits sequence and to start tracking around this pressure until a total of 12 
Table 1. Baseline Characteristics of IBS Patients With Bloating and Distention (B+D), and Bloating Alone (B)

\begin{tabular}{|c|c|c|c|c|c|c|c|c|c|}
\hline & \multicolumn{3}{|c|}{ IBS } & \multicolumn{3}{|c|}{ IBS-C } & \multicolumn{3}{|c|}{ IBS-D } \\
\hline & All & $B+D$ & B & All & $B+D$ & $\mathrm{~B}$ & All & $B+D$ & B \\
\hline \multicolumn{10}{|c|}{ Baseline characteristics } \\
\hline No. & 68 & $28(41 \%)$ & 40 (59\%) & 39 & 19 (49\%) & $20(51 \%)$ & 29 & $9(31 \%)$ & $20(69 \%)$ \\
\hline Age, yrs & $37.1(34.1,40.1)$ & $40.4(35.9,44.9) *$ & $34.9(31.0,38.8)$ & $36.1(32.1,40.2)$ & $40.1(34.1,46.0) *$ & $32.6(27.5,37.7)$ & $38.5(35.9,41.0)$ & $41.0(34.2,47.8)$ & $37.3(31.4,43.1)$ \\
\hline $\begin{array}{l}\text { Body mass } \\
\text { index, } \\
\mathrm{kg} / \mathrm{m}^{2}\end{array}$ & $25.0(23.9,26.2)$ & $25.8(23.9,27.7)$ & $24.5(23.0,26.0)$ & $25.1(23.7,26.5)$ & $25.7(23.4,27.9)$ & $24.5(22.7,26.4)$ & $25.0(22.9,27.0)$ & $26.1(22.1,30.1)$ & $24.5(22.1,26.8)$ \\
\hline Girth, cm & $83.3(80.1,86.5)$ & $87.1(82.1,92.1)^{*}$ & $81.0(76.9,85.1)$ & $83.1(78.8,87.3)$ & $86.0(79.7,92.2)$ & $80.7(74.9,86.4)$ & $83.7(78.5,88.9)$ & $90.1(81.7,98.5)$ & $81.3(75.2,87.4)$ \\
\hline $\begin{array}{c}\text { Bloating } \\
\text { score }\end{array}$ & $1.6(1.3,1.9)$ & $1.7(1.2,2.2)$ & $1.6(1.2,2.0)$ & $1.9(1.5,2.3)$ & $1.8(1.3,2.3)$ & $1.9(1.4,2.4)$ & $1.3(0.8,1.8)$ & $1.2(0.1,2.3)$ & $1.3(0.8,1.8)$ \\
\hline Anxiety & $9.0(7.9,9.0)$ & $8.6(7.1,10.1)$ & $9.2(7.7,10.7)$ & $8.5(7.2,9.9)$ & $8.3(6.3,10.3)$ & $8.7(6.9,10.6)$ & $9.6(7.9,11.3)$ & $9.3(7.4,11.1)$ & $9.7(7.4,12.1)$ \\
\hline Depression & $4.4(3.6,5.2)$ & $4.4(3.4,5.4)$ & $4.4(3.3,5.6)$ & $4.2(3.1,5.2)$ & $4.4(3.1,5.7)$ & $3.9(2.3,5.5)$ & $4.8(3.6,6.0)$ & $4.3(3.8,5.8)$ & $5.0(3.4,6.6)$ \\
\hline
\end{tabular}

Data expressed as mean $(95 \% \mathrm{CI})$. IBS, irritable bowel syndrome; IBS-C, IBS with constipation; IBS-D, IBS with diarrhea; B + D, bloating with distension; B, bloating alone; girth AUC, mean abdominal girth from the second hour of the study to the end of day 1 minus the mean girth for the first hour of the study; bloating AUC, mean bloating score from the second hour of the study to the end of day 1 minus the mean girth for the first hour of the study.

$* P<.1$ compared with patients who had bloating alone.

inflation trials had occurred. The sensory threshold was calculated as the average pressure over the series of tracking inflations rated as at least moderately painful. This procedure is designed to make the distention sequence unpredictable to the subject and to minimize response bias. ${ }^{13}$ At any time, both the patient and investigator could discontinue the inflation for any reason by pressing a "panic button."

\section{Data Analysis}

The subjective bloating scores at the start of the day and before retiring to bed on day 1 were recorded. Changes in abdominal girth and subjective bloating scores between subject groups were compared using independent $t$ tests and the ANOVA test. Within subject correlations between mean values and changes in abdominal girth and subjective bloating scores were performed using the Pearson test.

The following measurements were derived: (1) the sensory threshold for pain, calculated as the average pressure over the series of tracking inflations rated as at least moderate pain (score, 3); (2) rectal tone, calculated from the average bag volume while maintaining the pressure at the basal operating pressure for 15 minutes; ${ }^{14}$ (3) static compliance $\left(C_{\text {stat }}\right)$, calculated as the volume/pressure relationship at $20 \mathrm{~mm} \mathrm{Hg}{ }^{15}$ and (4) dynamic compliance $\left(C_{\mathrm{dyn}}\right)$, calculated from the slope of the compliance curve. ${ }^{16,17}$ In addition, each patient was classified as having a normally sensitive, hypersensitive, or hyposensitive rectum. This was based on the $95 \%$ reference range for the sensory threshold to moderate pain (score, 3) obtained from a panel of healthy volunteers. Patients having a rectal sensory threshold below the 2.5 percentile limit were defined as hypersensitive, those with a sensory threshold above the 97.5 percentile limit were defined as hyposensitive, and those with a sensory threshold between these limits were defined as normally sensitive. All data are expressed as mean and 95\% confidence intervals (CI). The study was reviewed and received approval by the South Manchester Local Research Ethics Committee.

\section{Results}

\section{Distending vs Nondistending Patients}

Compared with the 97.5 percentile of the diurnal change in abdominal girth (ie, distention) seen in healthy volunteers (ie, $2.8 \mathrm{~cm}$ ), ${ }^{1} 41 \%$ of all IBS patients, $49 \%$ of IBS-C patients, and $31 \%$ of IBS-D patients exhibited abdominal distention (Table 1). All subgroups had similar baseline characteristics, although IBS-C patients with concomitant bloating and distention $(\mathrm{B}+\mathrm{D})$ tended to be slightly older $(P=.07)$ than patients with bloating alone (B) (Table 1). Likewise, IBS patients overall with bloating and distention tended to be older $(P=.07)$ and have a greater girth at the beginning of the study $(P=$ .07) than those with bloating alone (Table 1).

Adjusting for the significant confounders of age, baseline girth, and anxiety, IBS patients with bloating alone had significantly lower pain thresholds $(P=.005)$ along with lower thresholds for first sensation $(P=.07)$ and desire to defecate $(P=.044)$ than patients with concomitant distention (Figure 1A). This reflected lower thresholds for pain $(P=.042)$ but not desire to defecate $(P=$ $.24)$ or first sensation $(P=.34)$ in IBS patients with constipation who had bloating alone compared with those who bloat and distend (Figure $1 B$ ) and lower thresholds for first sensation $(P=.08)$ and desire to defecate $(P=.029)$ but interestingly not pain $(P=.25)$ in IBS-D patients who had bloating alone compared with those who bloat and distend (Figure 1C). With respect to bloating, however, IBS-C patients (from the Lickert scale, B + D: mean, 1.79 [95\% CI: 1.37-2.21] vs B: mean, 0.53 [95\% CI: 0.13-0.93]; $P<.001)$ but not those with IBS-D (mean, 0.65 [95\% CI: -0.21 to 1.49$]$ vs mean, 1.18 [95\% CI: $0.70-1.76) ; P=.31$ ) who bloated and distended reported more bloating than those with bloating alone. Consequently, IBS patients overall who bloated and distended reported more bloating than those with bloating alone (mean, 1.52 [95\% CI: $1.10-1.94]$ vs mean, 0.86 [95\% CI: $0.50-1.22$ ], respectively; $P=.02$ ). 

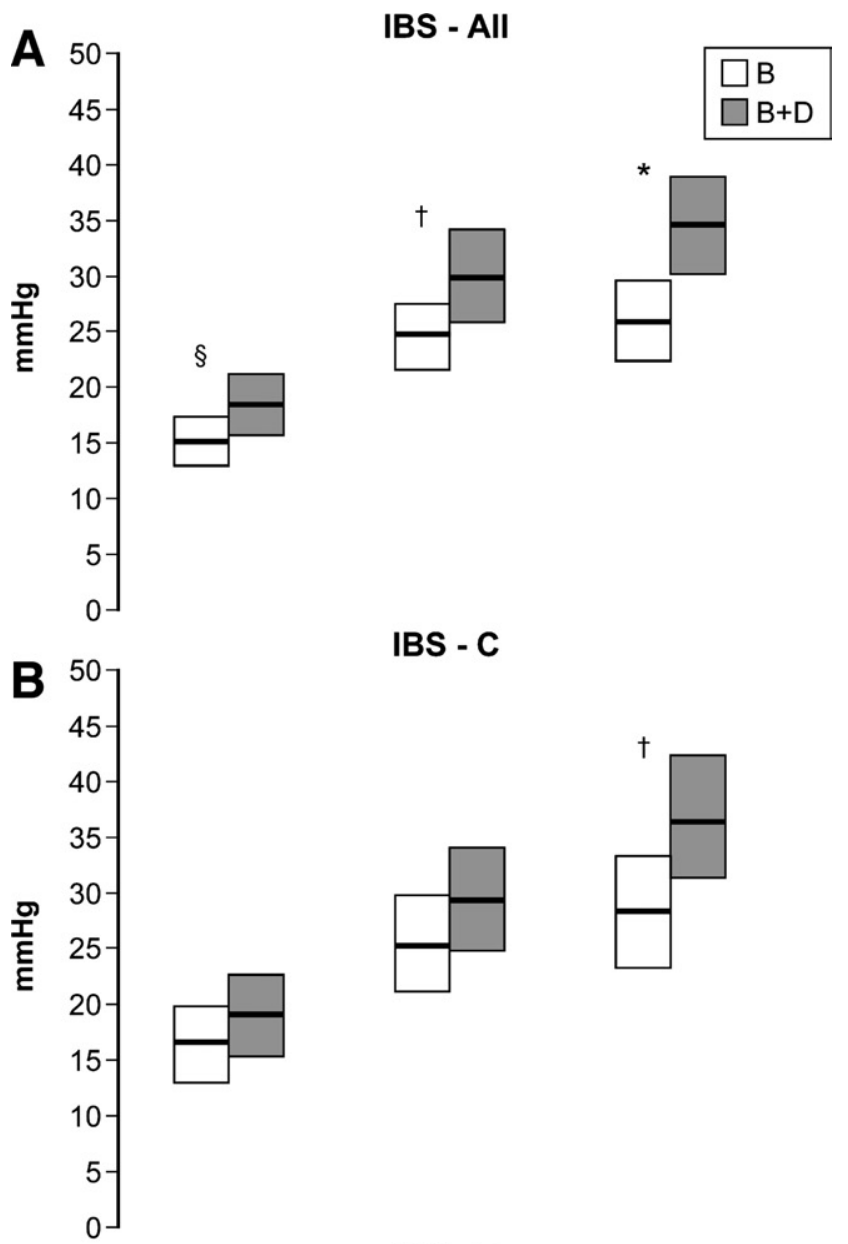

IBS - C

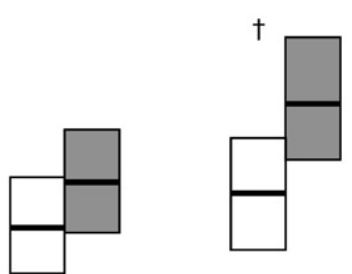

IBS - D
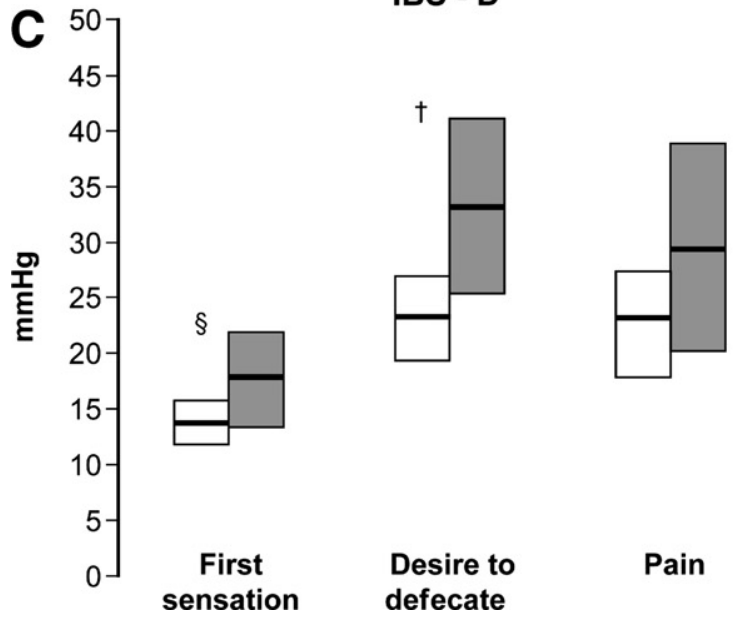

Figure 1. Comparison of first sensation, desire to defecate, and pain thresholds between nondistenders $(B)$ and distenders $(B+D)$ in IBS patients overall (IBS-AII), IBS patients with constipation (IBS-C), and IBS patients with diarrhea (IBS-D). Data expressed as mean and 95\% confidence interval. ${ }^{*} P<.01$ compared with panel $B+D$; ${ }^{\dagger} P<.05 \mathrm{com}$ pared with panel $B+D ; \S P<.1$ compared with panel $B+D$.

Furthermore, comparison of the numbers of patients exhibiting hyper-, normo-, and hyposensitivity revealed that a greater percentage of those IBS patients with bloating alone tended to be hypersensitive, whereas a greater percentage of those with bloating and distention tended to be hyposensitive to rectal distention $(P=.001)$. This was independent of the bowel habit, with more IBS-D $(P=.055)$ and IBS-C $(P=.034)$ patients with bloating alone hypersensitive compared with those who had associated distention, who were more likely to be hyposensitive (Table 2).

Adjusting for the significant confounders of baseline girth and body mass index (BMI) in the IBS group as a whole, there were no differences in either dynamic (mean, 8.74 [95\% CI: 7.18-10.30] mL/mm Hg vs mean, 8.67 [95\% CI: $6.89-10.45] \mathrm{mL} / \mathrm{mm} \mathrm{Hg}$, respectively; $P=.95$ ), static compliance (mean, 5.33 [95\% CI: 4.21-6.45] mL/mm Hg vs mean, 5.96 [95\% CI: $5.08-6.84] \mathrm{mL} / \mathrm{mm} \mathrm{Hg}$, respectively; $P=.39$ ), or tone (mean, 73.5 [95\% CI: $57.78-$ 89.22] $\mathrm{mL}$ vs mean, 91.60 [95\% CI: $72.26-110.94] \mathrm{mL}$, respectively; $P=.25$ ) between those IBS patients who distended and those who did not. Likewise, there were no differences between the IBS-C and IBS-D subgroups.

It is noteworthy that, of the patients who bloated and distended, significantly more patients had undergone hysterectomy $(32 \%)$ than those patients with bloating alone $(3 \% ; P=.015)$. In addition, hysterectomized patients tended to have higher pain thresholds (mean, 35.9 [26.3-44.5] $\mathrm{mm} \mathrm{Hg}$ vs mean, 27.5 [95\% CI: 24.1-30.9] $\mathrm{mm} \mathrm{Hg} P=.135)$ and greater abdominal distention (mean, 5.51 [95\% CI: 3.50-7.52] cm vs mean, 2.00 [95\% CI: $1.26-2.74] \mathrm{cm}$, respectively; $P=.011)$ but not dynamic (mean, 8.76 [95\% CI: $4.76-12.76] \mathrm{mL} / \mathrm{mm} \mathrm{Hg}$ vs mean, 8.02 [95\% CI: $6.64-9.40] \mathrm{mL} / \mathrm{mm} \mathrm{Hg}$ ) or static (mean, 4.21 [95\% CI: $2.15-6.27] \mathrm{mL} / \mathrm{mm} \mathrm{Hg}$ vs mean, 5.83 [95\% CI: $4.99-6.67] \mathrm{mL} / \mathrm{mm} \mathrm{Hg}$ ) compliance compared with nonhysterectomized patients.

\section{Hyper- vs Normo- vs Hyposensitive Patients}

Compared with a $95 \%$ reference range for pain threshold in healthy volunteers $(24-38 \mathrm{~mm} \mathrm{Hg}),{ }^{18} 43 \%$ of IBS patients were hypersensitive, $35 \%$ normosensitive, and $22 \%$ hyposensitive. This reflected more patients with IBS-D being hypersensitive (62\%) than either normo$(24 \%)$ or hyposensitive $(14 \%)(P<.004)$ but a similar

Table 2. Comparison of Numbers of Hyper-, Normo-, and Hyposensitive Patients Who Concomitantly Bloat and Distend or Bloat Alone

\begin{tabular}{|c|c|c|c|c|}
\hline & & \multicolumn{2}{|c|}{ No. (\%) with $B+D$ or $B$} & \multirow[b]{2}{*}{ Chi-square test } \\
\hline & & $B+D$ & B & \\
\hline \multirow[t]{3}{*}{ IBS } & Hyper- & $5(17 \%)$ & $24(83 \%)$ & \\
\hline & Normo- & $12(50 \%)$ & $12(50 \%)$ & \\
\hline & Hypo- & $11(73 \%)$ & $4(27 \%)$ & $P=.001$ \\
\hline \multirow[t]{3}{*}{ IBS-C } & Hyper- & $2(18 \%)$ & $9(82 \%)$ & \\
\hline & Normo- & $9(53 \%)$ & $8(47 \%)$ & \\
\hline & Hypo- & $8(73 \%)$ & $3(27 \%)$ & $P=.034$ \\
\hline \multirow[t]{3}{*}{ IBS-D } & Hyper- & $3(17 \%)$ & $15(83 \%)$ & \\
\hline & Normo- & $3(43 \%)$ & $4(57 \%)$ & \\
\hline & Hypo- & $3(75 \%)$ & $1(25 \%)$ & $P=.055$ \\
\hline
\end{tabular}


Table 3. Baseline Characteristics of IBS Patients Who Were Either Hyper-, Normo-, or Hyposensitive to Rectal Distention

\begin{tabular}{|c|c|c|c|c|c|c|c|c|c|}
\hline & \multicolumn{3}{|c|}{ IBS-all $(n=68)$} & \multicolumn{3}{|c|}{ IBS-C $(n=39)$} & \multicolumn{3}{|c|}{ IBS-D $(n=29)$} \\
\hline & Hyper- & Normo- & Hypo- & Hyper- & Normo- & Нypo- & Hyper- & Normo- & Hypo- \\
\hline \multicolumn{10}{|c|}{ Baseline characteristics } \\
\hline No. & 29 (43\%) & 24 (35\%) & $15(22 \%)$ & $11(28 \%)$ & 17 (44\%) & $11(28 \%)$ & $18(62 \%)$ & $7(24 \%) *$ & $4(14 \%) *$ \\
\hline Age, y & $36.3(31.5,41.1)$ & $34.8(30.1,39.5)$ & $42.1(34.9,49.2)$ & $35.1(25.5,44.6)$ & $32.9(28.1,38.7)$ & $41.9(32.5,51.3)$ & $37.1(31.1,43.1)$ & $40.0(25.8,54.2)$ & $42.5(24.4,60.6)$ \\
\hline $\begin{array}{l}\text { Body mass } \\
\text { index, } \\
\mathrm{kg} / \mathrm{m}^{2}\end{array}$ & $22.9(21.2,24.5)$ & $26.7(24.9,28.5)^{*}$ & $26.5(23.6,29.3)^{+}$ & $22.8(19.6,26.1)$ & $26.0(23.9,28.0)$ & $26.0(22.9,29.1)$ & $22.9(20.8,25.0)$ & $28.6(23.5,33.6)^{+}$ & $27.90(11.7,44.1)$ \\
\hline Girth, cm & $79.3(75.0,83.5)$ & $85.1(78.7,91.4)$ & $88.6(80.9,96.3)$ & $77.9(68.8,86.9)$ & $84.3(77.1,91.6)$ & $86.4(78.3,94.5)$ & $80.2(75.3,85.1)$ & $87.8(63.6,112.1)$ & $95.8(53.4,138.2)$ \\
\hline $\begin{array}{c}\text { Bloating } \\
\text { score }\end{array}$ & $1.8(1.3,2.2)$ & $1.4(0.8,2.0)$ & $1.7(1.1,2.3)$ & $2.3(1.4,3.1)$ & $1.5(0.9,2.1)$ & $1.9(1.2,2.6)$ & $1.4(0.9,1.9)$ & $1.0(0,5.3)$ & $1.0(0,3.5)$ \\
\hline Anxiety & $10.5(8.7,12.3)$ & $7.7(6.1,9.3)^{\dagger}$ & $7.9(5.8,10.0)$ & $10.5(7.1,13.8)$ & $8.2(6.3,10.1)$ & $7.1(4.4,9.8)$ & $10.5(8.1,12.9)$ & $6.0(3.1,8.9)$ & $10.0(6.6,13.4)$ \\
\hline Depression & $5.1(3.6,6.7)$ & $3.6(2.7,4.6)$ & $4.1(2.6,5.6)$ & $5.7(2.5,8.9)$ & $3.4(2.4,4.3)$ & $3.8(2.0,5.7)$ & $4.8(3.0,6.5)$ & $4.6(0.9,8.3)$ & $5.0(0.7,9.3)$ \\
\hline
\end{tabular}

Data expressed as mean $(95 \% \mathrm{Cl})$. Hyper, hypersensitive patients; Normo, normally sensitive patients; Hypo, hyposensitive patients.

$* P<.05$.

$\dagger P<.1$ compared with hypersensitive

distribution among IBS-C patients being hyper- (28\%), normo- (44\%), and hyposensitive $(28 \%)(P=.397)$ (Table 2).

Examination of the baseline characteristics revealed no significant differences in age, girth, bloating score, or depression level among the various patient subgroups, although BMI did tend to be lower in the hypersensitive IBS-D patients compared with those who were normally sensitive $(P<.10)$ and lower in hypersensitive IBS patients overall compared with normo- $(P<.05)$ and hyposensitive $(P<.10)$ patients. Furthermore, anxiety levels tended to be higher in the hypersensitive IBS patients overall compared with those who were normally sensitive $(P<.10)$ (Table 3).

Adjusting for the significant confounder of age, there were significant differences in the degree of abdominal distention seen among the hyper-, normo-, and hyposensitive IBS-C patients $(P=.002)$, such that hyposensitive IBS-C patients distended significantly more than their hyper- $(P=.002)$ and normosensitive $(P=.053)$ counterparts (Figure 2). Abdominal distention was substantially more variable for the various sensitivity subgroupings of the IBS-D patients, and, consequently, no differences were observed among the 3 subgroups $(P=$ .22). However, in IBS patients overall, there was a statistical difference among the 3 sensitivity subgroups $(P<$ .001 ), and abdominal distention was significantly greater in hyposensitive compared with hypersensitive patients $(P<.001)$ (Figure 2).

Adjusting for the significant confounders of age and baseline girth, there were significant differences in the severity of bloating reported among the hyper- (mean, 0.45 [95\% CI: -0.08 to 0.97]), normo- (mean, 1.67 [95\% CI: 1.07-2.27]), and hyposensitive (mean, 1.21 [95\% CI: $0.52-1.90])$ IBS-C patients $(P=.009)$; such that those who were normosensitive reported significantly more bloating than those who were hypersensitive $(P=.007)$. There were no differences in bloating scores among the hyper-, normo-, and hyposensitive subgroups for either the IBS-D patients (mean, 1.19 [95\% CI: 0.52-1.88]; mean, 0.75 [95\% CI: -1.05 to 2.56 ]; and mean, 1.06 [95\% CI:
$0.55-1.56])$, respectively $(P=.75)$ or IBS patients overall (mean, 0.90 [95\% CI: 0.44-1.36]; mean, 1.48 (95\% CI: 0.95-2.03]; and mean, 1.08 [95\% CI: 0.55-1.61]), respectively $(P=.10)$.

Adjusting for the significant confounder of baseline girth, there was a significant difference in dynamic compliance among the hyper-, normo-, and hyposensitive IBS patients $(P=.034)$ such that normosensitive IBS patients had a greater rectal compliance compared with those who were hyposensitive (mean, $10.03 \mathrm{~mL} / \mathrm{mmHg}$ [95\% CI: 5.67-11.01] vs mean, $7.13 \mathrm{~mL} / \mathrm{mmHg}$ [95\% CI: 5.15 9.12]), respectively; $(P=.03)$. Examination of the indi-

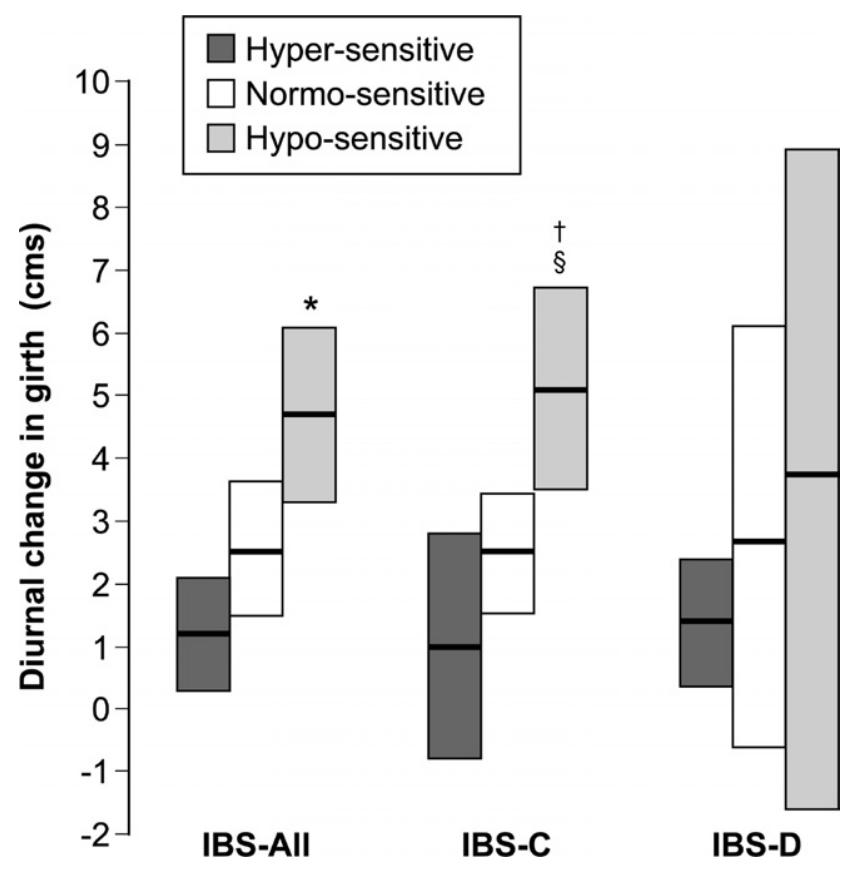

Figure 2. Comparison of diurnal change in girth (distention) between hypersensitive, normosensitive, and hyposensitive patients in IBS patients overall (IBS-all), IBS patients with constipation (IBS-C), and IBS patients with diarrhea (IBS-D). Data expressed as mean and 95\% confidence interval. ${ }^{\star} P<.001$ compared with hypersensitive; ${ }^{\dagger} P<.01$ compared with hypersensitive; $\$ P<.1$ compared with normosensitive. 
vidual bowel habit subgroups revealed no significant differences among the 3 visceral sensitivity subgroups (data not supplied). Likewise, there were no differences in static compliance or tone among the 3 visceral sensitivity subgroups for either the IBS-C or IBS-D patients or patients overall (data not supplied).

More of the hyposensitive patients (38\%) had undergone previous hysterectomy than either hypersensitive $(8 \% ; P=.034)$ and normosensitive $(8 \% ; P=.16)$ patients. There was no difference between hyper- and normosensitive patients.

\section{Correlations}

No significant correlations were observed between either bloating or distention and age, initial girth, BMI, anxiety, or depression in IBS patients overall, as well as in IBS-C and IBS-D patients. However, bloating severity did correlate with the degree of abdominal distention in IBS-C $(r=0.37, P<.05)$ but not IBS-D $(r=-0.088, P$ $=.9$ ) patients. Furthermore, the degree of abdominal distention correlated with pain threshold in both IBS-C $(r=0.60, P<.001)$ and IBS-D $(r=0.35, P=.06)$ patients and, consequently, IBS patients overall $(r=0.51$, $P<.001)$. No correlation was found between the severity of bloating and sensory threshold for pain in any of the patient groups.

\section{Discussion}

This study has clearly shown that there is a relationship between the symptom of bloating without distention and the presence of visceral hypersensitivity. In contrast, when an actual increase in girth is experienced during the course of the day, this is more likely to be associated with visceral hyposensitivity.

As already mentioned, there have been a number of postulated mechanisms for these phenomena, although some, such as deliberate protrusion of the abdomen or excessive gas, have been investigated and proved to be unlikely. ${ }^{19,20}$ However, the Barcelona group has demonstrated that, even if there are not excessive amounts of gas present in patients with IBS, the handling of infused gas is abnormal in this condition ${ }^{21,22}$ and that this might be controlled by tonic contraction of the gut. ${ }^{23}$ In addition, it seems likely that this abnormality may in some way contribute to the symptom of bloating, and there is evidence to suggest that the abnormality is more likely to reside in the small bowel rather than in the colon. ${ }^{24}$ It also seems reasonable to assume that there is the capacity for some form of accommodation reflex within the anterior abdominal wall to allow for events such as the ingestion of a meal or the expansion of the pregnant uterus. Abdominal electromyographic studies in response to events such lying, standing, and performing voluntary contraction of the abdominal wall have not revealed any differences between IBS patients and controls. ${ }^{25}$ In contrast, when gas is infused into the colon, it has been shown that the normal electromyographic response is for increased activity, whereas in IBS there is a failure of tonic contraction of the abdominal wall accompanied by paradoxical relaxation of the internal oblique muscles. ${ }^{26}$ Thus, it appears that bloating or distention, even on their own, is unlikely to have a single cause, and a multifactorial basis for the cause of both of these symptoms is a much more plausible hypothesis.

Diarrhea-predominant IBS has been shown to be more commonly associated with visceral hypersensitivity, ${ }^{8}$ and, therefore, the present finding of a relationship between this sensory abnormality and bloating without distention is in accord with our previous observation that bloating alone is more common in patients with diarrhea. ${ }^{1}$ These findings support the notion that there may be more of a sensory component to the phenomenon of bloating compared with distention. Although our assessment of visceral sensation was confined to the rectum, there is evidence that, when present, visceral hypersensitivity in patients with IBS may be much more widely expressed in the gut and can be shown to affect both the small intestine as well as the colon. ${ }^{27}$ Thus, in the presence of visceral hypersensitivity, perturbation of the mucosa at different levels from a variety of different stimuli could theoretically result in the sensation of bloating. It has also been shown that the perimenstrual phase of the menstrual cycle is associated with the highest levels of rectal sensitivity, ${ }^{15}$ and our findings might even partly explain why women complain of bloating at this time of their cycle.

We have previously shown that, in a cohort of IBS patients, all of whom suffered from bloating, distention can occur in both the diarrhea- and constipation-predominant subgroups, although it appears to be more pronounced in those suffering from constipation. ${ }^{1}$ Furthermore, there was a strong correlation between bloating scores and distention in those subjects with constipation, whereas this does not appear to be the case in those individuals with diarrhea. ${ }^{1}$ This is in accord with clinical studies in which patients appear to report a greater increase in girth with constipation. ${ }^{1,2}$ It is also of interest that pharmacologic induction of constipation with loperamide can induce distention even in normal individuals, in whom, presumably, visceral sensation is not disordered in any way. ${ }^{28}$ As previously mentioned, hyposensitivity occurs in a proportion of individuals suffering from constipation-predominant IBS, and, therefore, it is noteworthy that in the present study this was the very sensation abnormality that was associated with distention. Furthermore, the higher the pain thresholds experienced by the IBS patients, the more they distended during the course of the day. This raises the possibly that, rather than being mediated by visceral hypersensitivity, distention may have more of a mechanical component, possibly as a result of an accumulation of fecal material within the bowel. Furthermore, it is tempting to speculate 
that when the colon is stretched by fecal loading it might initiate the accommodation reflex previously alluded to, resulting in relaxation of the anterior abdominal musculature leading to an increase in abdominal girth.

Interestingly, patients who had undergone hysterectomy exhibited both rectal hyposensitivity and abdominal distention. Although some studies have shown a high incidence of bloating ${ }^{29}$ and rectal hyposensitivity ${ }^{30}$ in women following hysterectomy, others have shown either an increase ${ }^{31}$ or no difference ${ }^{32}$ in rectal sensitivity. Thus, the interpretation of this data is unclear, especially because the majority of our patients exhibiting abdominal distention (68\%) and rectal hyposensitivity (63\%) had not undergone a hysterectomy.

The treatment of IBS is notoriously unsatisfactory, and, of the various symptoms of which patients complain, bloating and distention have always been especially hard to manage, largely because of a lack of any understanding of their pathophysiologic basis. Hopefully, as the various mechanisms involved become better understood, management strategies can be developed that take account of these abnormalities. For instance, now that we know that the handling of an exogenous gas load is often abnormal in IBS, it makes it reasonable to advise patients to avoid carbonated drinks or foods as well as additives that can lead to excessive gas production. Similarly, our current and previous observations on the possible relationship of constipation with distention in particular suggest that relieving constipation with either a laxative or a prokinetic might be expected to lead to an improvement in this symptom. This possibly explains why a reduction of bloating scores has been a consistent finding in trials of the prokinetic tegaserod ${ }^{33-35}$ because, although actual physical distention was not recorded in any of these studies, the symptom of bloating could be acting as a marker for a change of girth. The observation that visceral hypersensitivity appears to contribute more to the sensation of bloating rather than distention indicates that this symptom might be amenable to an approach based on modifying this sensory abnormality. Unfortunately, there are not a large number of pharmacologic agents known to reliably reduce hypersensitivity, although pregabalin has recently been shown to have this effect in patients with IBS. ${ }^{36}$ In addition, it has been suggested that some of the beneficial effects of tricyclic antidepressants in IBS might be mediated by an effect on visceral hypersensitivity, ${ }^{37}$ although not all studies support this view. However, neither tricyclics nor pregabalin has been formally evaluated in patients with bloating. Hypnotherapy consistently reduces symptomatic bloating, ${ }^{38-40}$ although its effect on abdominal distention has not been objectively assessed. It is therefore of interest that it has been shown that this technique can reduce visceral hypersensitivity ${ }^{18}$ as well as modulating the central processing of painful stimuli. ${ }^{41}$ With respect to any future research in relation to treatment or of a more mechanistic nature, it will be essential that bloating and distention are regarded as potentially separate entities, although patients are likely to continue using the descriptor bloating to describe both phenomena. Such an approach should avoid some of the confusion that has occurred in trying to interpret data from past studies.

\section{References}

1. Houghton LA, Lea R, Agrawal A, et al. Relationship of abdominal bloating to distention in irritable bowel syndrome and effect of bowel habit. Gastroenterology 2006;131:1003-1010.

2. Chang L, Lee OY, Naliboff $B$, et al. Sensation of bloating and visible abdominal distention in patients with irritable bowel syndrome. Am J Gastroenterol 2001;96:3341-3347.

3. Lembo T, Naliboff B, Munakata J, et al. Symptoms and visceral perception in patients with pain-predominant irritable bowel syndrome. Am J Gastroenterol 1999;94:1320-1326.

4. Lewis MJ, Reilly B, Houghton LA, et al. Ambulatory abdominal inductance plethysmography: towards objective assessment of abdominal distension in irritable bowel syndrome. Gut 2001;48: 216-220.

5. Reilly BP, Bolton M, Lewis MJ, et al. A device for 24-hour ambulatory monitoring of abdominal girth using inductive plethysmography. Physiol Meas 2002;23:661-670.

6. Houghton LA, Whorwell PJ. Towards a better understanding of abdominal bloating and distension in functional gastrointestinal disorders. Neurogastroenterol Motil 2005;17:500-511.

7. Azpiroz F, Malagelada JR. Abdominal bloating. Gastroenterology 2005;129:1060-1078.

8. Mertz H, Naliboff $\mathrm{B}$, Munakata J, et al. Altered rectal perception is a biological marker of patients with irritable bowel syndrome. Gastroenterology 1995;109:40-52.

9. Bouin M, Plourde V, Boivin M, et al. Rectal distention testing in patients with irritable bowel syndrome: sensitivity, specificity, and predictive values of pain sensory thresholds. Gastroenterology 2002;122:1771-1777.

10. Hammonds R, Houghton LA, Whorwell PJ. Urge and no-urge constipation predominant irritable bowel syndrome (IBS): sensory dysfunction of the whole gut. Gastroenterology 2000;118(Suppl 2):830.

11. Harraf F, Schmulson M, Saba L, et al. Sub-types of constipation predominant irritable bowel syndrome based on rectal perception. Gut 1998;43:388-394.

12. Thompson WG, Longstreth GF, Drossman DA, et al. Functional bowel disorders and functional abdominal pain. Gut 1999; 45(Suppl 2):1143-1147.

13. Whitehead WE, Delvaux M, and the Working Team. Standardization of barostat procedures for testing smooth muscle tone and sensory thresholds in the gastrointestinal tract. Dig Dis Sci 1997;42:223-241.

14. Thumshirn M, Camilleri M, Choi M-G, et al. Modulation of gastric sensory and motor functions by nitrergic and $\alpha-2$ adrenergic agents in humans. Gastroenterology 1999;116:573-585.

15. Houghton LA, Lea $R$, Jackson $N$, et al. The menstrual cycle affects rectal sensitivity in patients with irritable bowel syndrome but not healthy volunteers. Gut 2002;50:471-474.

16. Lembo T, Munakata J, Mertz H, et al. Evidence for hypersensitivity of lumbar splanchnic afferents in irritable bowel syndrome. Gastroenterology 1994;107:1686-1696.

17. Jackson NA, Houghton LA, Whorwell PJ, et al. Does the menstrual cycle affect anorectal physiology?. Dig Dis Sci 1994;39:26072611.

18. Lea R, Houghton LA, Calvert E, et al. Gut-focused hypnotherapy normalizes disordered rectal sensitivity in patients with irritable bowel syndrome. Aliment Pharmacol Ther 2003;17:635-642. 
19. Lasser RB, Bond JH, Levitt MD. Role of intestinal gas in functional abdominal pain. N Engl J Med 1975;293:524-526.

20. Maxton DG, Martin DF, Whorwell PJ, et al. Abdominal distension in female patients with irritable bowel syndrome: exploration of possible mechanisms. Gut 1991;32:662-664.

21. Serra J, Azpiroz F, Malagelada JR. Impaired transit and tolerance of intestinal gas in the irritable bowel syndrome. Gut 2001;48: 14-19.

22. Serra J, Azpiroz F, Malagelada JR. Mechanisms of intestinal gas retention in humans: impaired propulsion versus obstructed evacuation. Am J Physiol Gastrointest Liver Physiol 2001;281: G138-G143.

23. Tremolaterra F, Villoria A, Serra J, et al. Intestinal tone and gas motion. Neurogastroenterol Motil 2006;18:905-910.

24. Salvioli B, Serra J, Azpiroz F, et al. Origin of gas retention and symptoms in patients with bloating. Gastroenterology 2005;128: 574-579.

25. McManis PG, Newall D, Talley NJ. Abdominal wall muscle activity in irritable bowel syndrome with bloating. Am J Gastroenterol 2001;96:1139-1142.

26. Tremolaterra F, Villoria A, Azpiroz F, et al. Impaired viscerosomatic reflexes and abdominal-wall dystony associated with bloating. Gastroenterology 2006;130:1062-1068.

27. Francis $\mathrm{CY}$, Houghton LA, Whorwell PJ, et al. Enhanced sensitivity of the whole gut in patients with irritable bowel syndrome (abstr). Gastroenterology 1995;108:601.

28. Marcus SN, Heaton KW. Irritable bowel type symptoms in spontaneous and induced constipation. Gut 1987;28:156-159.

29. Heaton KW, Parker D, Cripps H. Bowel function and irritable bowel syndrome after hysterectomy and cholecystectomy-a population-based study. Gut 1993;34:1108-1111.

30. Varma JS, Smith AN. Abnormalities of colo-rectal function in intractable constipation following hysterectomy. Gut 1985;26: 581-582.

31. Prior A, Stanley KM, Smith AR, et al. Effect of hysterectomy on anorectal and urethrovesical physiology. Gut 1992;33:264-267.

32. Roe AM, Bartolo DCC, McMortensen NJ. Slow transit constipation. Comparison between patients with or without previous hysterectomy. Dig Dis Sci 1988;33:1159-1163.

33. Muller-Lissner SA, Fumagalli I, et al. Tegaserod, a 5-HT(4) receptor partial agonist, relieves symptoms in irritable bowel syndrome patients with abdominal pain, bloating and constipation. Aliment Pharmacol Ther 2001;15:1655-1666.
34. Novick J, Miner P, Krause R, et al. A randomized, double-blind, placebo-controlled trial of tegaserod in female patients suffering from irritable bowel syndrome with constipation. Aliment Pharmacol Ther 2002;16:1877-1888.

35. Whorwell PJ, Ruegg P, Earnest D, et al. Tegaserod significantly improves bloating in female irritable bowel syndrome patients with constipation. Gastroenterology 2004;126:A643.

36. Houghton LA, Fell C, Whorwell PJ, et al. Effect of a secondgeneration $\alpha 2 \delta$ ligand (pregabalin) on visceral sensation in hypersensitive patients with irritable bowel syndrome. Gut 2007; 56:1218-1225.

37. Poitras P, Riberdy Poitras M, Plourde V, et al. Evolution of visceral sensitivity in patients with irritable bowel syndrome. Dig Dis Sci 2002;47:914-920.

38. Whorwell PJ, Prior A, Faragher EB. Controlled trial of hypnotherapy in the treatment of severe refractory irritable-bowel syndrome. Lancet 1984;2:1232-1234.

39. Gonsalkorale WM, Houghton LA, Whorwell PJ. Hypnotherapy in irritable bowel syndrome: a large scale audit of a clinical service with an examination of factors influencing responsiveness. Am J Gastroenterol 2002;97:954-961.

40. Gonsalkorale WM, Miller V, Afzal A, et al. Long-term benefits of hypnotherapy for irritable bowel syndrome. Gut 2003;52:16231629.

41. Rainville P, Duncan GH, Price DD, et al. Pain affect encoded in human anterior cingulate but not somatosensory cortex. Science 1997;277:968-971.

Received August 8, 2007. Accepted February 28, 2008.

Address requests for reprints to: L. A. Houghton, MD, Neurogastroenterology Unit, University of Manchester, Wythenshawe Hospital, Southmoor Road, Manchester M23 9LR, United Kingdom. e-mail: Lesley.Houghton@manchester.ac.uk; fax: (44) 1612914184.

Supported in part by an educational grant from Novartis Pharmaceuticals, Basal, Switzerland.

Conflicts of interest and financial disclosure: Professor P. J. Whorwell and $\mathrm{Dr}$ L. A. Houghton have received remuneration for advice, and their department has also received financial support from Novartis Pharmaceuticals, GlaxoSmithKline, Pfizer, Solvay Pharmaceuticals, Rotta Research, Procter and Gamble, Danone Research, Astellas Pharma, and Tillots Pharma. Dr A. Agrawal, Dr R. Lea, J. Morris, and B. Reilly have no conflicts of interest to disclose. 